Results Prior to $N$ gonorrhoeae inoculation, mice with a pre-existing chlamydial infection had decreased expression of TLR4 and antimicrobial peptide (CRAMP, SLPI) genes. Consistent with the finding of decreased TLR4expression in coinfected mice, markers of inflammation (TLR2, TNF $\alpha$, IL-1B, platelet activating factor receptor [Pafr], and IL-23 $\alpha$ ) were up-regulated only in mice infected with $N$ gonorrhoeae alone. A significantly lower percentage of TLR4expressing epithelial cells was detected in vaginal swabs from chlamydia-infected wild-type mice prior to gonococcal challenge, and importantly, chlamydial infection did not enhance $N$ gonorrhoeae infection of TLR4 mutant mice.

Conclusions These data suggest the host response to chlamydial infection creates an environment that is less protective against gonococcal infection by down-regulating the expression of TLR4 and antimicrobial peptides. This work therefore further illuminates the basis of this interesting consequence of coinfection and may also help direct the development of immunomodulatory therapies against this common coinfection and its consequences on reproductive health.

\section{4-S2.02 SEROPREVALENCE OF NOVEL IMMUNOGENS OF CHLAMYDIA TRACHOMATIS AND THEIR CYTOKINE RESPONSE IN PBMC CELLS UNDER IN VITRO CONDITIONS}

doi:10.1136/sextrans-2011-050109.146

D Saluja, A Patel, P Mishra, D Sachdev. University Of Delhi, Delhi, India

Background Chlamydia trachomatis (CT) is an obligate intracellular parasite which causes STD and trachoma. Despite major research into Chlamydial pathogenesis and host immune responses, immuno-protection has been hampered by the incomplete understanding of protective immunity in the genital tract. Characterised vaccine candidates in general have shown variable efficacy ranging from no protection to partial protection. It is therefore a research priority to identify novel Chlamydial antigens that may elicit protective immune responses.

Objectives The goal of the present study was to assess the seroprevalence to pkn1and DNA j following natural CT infection in human. The prospects of pkn1 as a Type third secretion substrate and DNA j a non surface Chlamydial protein as potential antigen, prompted us to explore the immunogenic potential of both protein. Methods pkn, DNA j and ompA were cloned in bacterial expression vector pTrcHis. Ni+-NTA affinity chromatography was used to purify the recombinant proteins. Antigenic stretches of Pkn1, DNA j and OmpA were identified using Bcepred web server, designed for identification of subunit vaccine candidate by Bioinformatics Centre of IMTECH Chandigarh, India. To validate the bioinformatics based analysis, sera of human patient were used to determine seroreactivity of pkn1 and DNA j proteins. OmpA was used as a positive control during the study.

Results Present study showed a high seroprevalence of antibodies against Pkn1 and OmpA $(p<0.001)$ in sera of humans infected with CT. while, no antibodies were observed for DNA j. Our studies have shown an association between release of TNF- $\alpha$ and IFN-y levels upon stimulation of PBMC with $\mathrm{Pkn} 1$ and OmpA. Cytokine expression profiling (IL-1ß, TNF- $\alpha$, IL-2, IL10 and IFN-y) of Human PBMCs in response to Pkn1 stimulation demonstrate for the first time that Pkn1 is a novel immunodominant Chlamydial antigen that is capable of influencing both Th1 and Th2 immune responses by stimulating the release of both Humoural and Cell-mediated regulatory cytokines.

Conclusions Our study demonstrated strong serological responses to Pkn1 and major outer membrane in natural human infection suggesting the role of pkn1 in immune response. Studies are in progress to check how the immunomodulation by Pkn1 alters hostpathogen interactions.

\section{4-S2.03 AN ANTI-ADHESIVE APPROACH TO PREVENTION OF C TRACHOMATIS INFECTION}

doi:10.1136/sextrans-2011-050109.147

L A Campbell, S V Zidal, D Patton, Cho-chou Kuo. University of Washington Seattle, USA

Background We have reported that the major outer membrane of $C$ trachomatis is glycosylated and the glycan is a high mannose oligosaccharide. Cumulative studies have demonstrated that the glycan is important in attachment and infectivity through binding to the mannose receptor (MR). Glycan removal decreases infectivity in vitro and in vivo and simultaneous administration of mannan, a ligand of the $M R$, abrogates $C$ trachomatis infection in a mouse model of pneumonitis. Thus, we are investigating the feasibility of an anti-adhesive therapy to prevent $C$ trachomatis infection by identifying oligosaccharides that are effective in inhibiting infection in vitro and testing their efficacy in a mouse model of genital tract infection.

Methods HeLa 229 cell monolayers were pretreated with serial concentrations of oligosaccharides prior to infection with $C$ trachomatis. Neutralisation of infectivity was scored as $>50 \%$ inhibition. For animal experiments, 8-week-old Swiss Webster female mice were primed with subcutaneous injections of Depo-Provera 1 week prior to challenge. Subsequently, mice were inoculated intravaginally with carbohydrates or PBS ( $n=5$ mice per group) 30 min prior to infection with $C$ trachomatis. Vaginal swab samples were obtained at 24, 48, and $72 \mathrm{~h}$. post-infection, at peak times of shedding. Statistical significance was determined by the Student's t test. Results Carbohydrates have been tested in vitro in hapten inhibition experiments against three serovars (D, E, F) most frequently isolated from genital tract infection. At the highest concentration tested, 4nitrophenyl- $\alpha$-D-mannopyranoside inhibited infectivity by $91 \%-$ $92 \%$; $\boldsymbol{\alpha}$-D-mannose-PAA from 77 to $93 \%$; hen ovalbumin by $85 \%-$ $89 \%$; mannan by $77 \%-83 \%$; and the high mannose fraction prepared from ovalbumin by $59 \%-98 \%$. To determine efficacy in vivo, Swiss Webster mice were inoculated with different concentrations of inhibitors. Of those tested thus far, shedding of organism was significantly decreased $(p<0.05)$. The maximum inhibitions observed were: 4 nitrophenyl- $\alpha$-D-mannopyranoside (86\%), $\alpha$-D-mannose-PAA (81\%), mannan (93\%), and the high mannose fraction from ovalbumin $(94 \%)$. Conclusions These preliminary studies suggest the potential feasibility for developing an "anti-adhesive therapy" as an alternate topical microbicide approach to prevent $C$ trachomatis genital tract infection.

\section{4-S2.04 CAN A CERVICAL BARRIER PREVENT CHLAMYDIA INFECTION IN THE PIGTAILED MACAQUE CERVICAL CHALLENGE MODEL?}

doi:10.1136/sextrans-2011-050109.148

${ }^{1} \mathrm{D}$ Patton, ${ }^{1} \mathrm{Y}$ Cosgrove Sweeney, ${ }^{2} \mathrm{~T}$ Moench. ${ }^{1}$ University of Washington, Seattle, USA; ${ }^{2}$ ReProtect, Inc, Baltimore, USA

Background Numerous microbicides that inactivate Chlamydia trachomatis in vitro have failed to prevent transmission of this pathogen in the pigtailed macaque cervical challenge model. Since $C$ trachomatis replicates in endocervical columnar epithelium but not in the squamous vaginal epithelium, we tested whether a cervical barrier would improve protection when used in conjunction with an otherwise non-protective microbicide. 
Abstract 04-S2.04 Table 1 CxBar

\begin{tabular}{|c|c|c|c|}
\hline \multirow[t]{2}{*}{ No Protection } & \multicolumn{3}{|c|}{ Protection } \\
\hline & $\begin{array}{l}\text { Working definition of confirmed infection: } \\
\text { Positive NAAT AND Positive culture and } \\
\text { Positive } \lg G / \lg M>1: 16\end{array}$ & $\begin{array}{l}\text { Working definition of probable infection: } \\
\text { positive } N A A T \text { and positive culture \& } \lg G / \\
\lg M<1: 32 \text { OR negative culture \& negative } \\
\text { culture } \lg G / \lg M>1: 32\end{array}$ & $\begin{array}{l}\text { Working definition of no infection: nega- } \\
\text { tive or self-limited NAAT AND negative } \\
\text { culture AND } \lg G / \lg M<1: 32\end{array}$ \\
\hline Barrier with buffergel & 2 & 2 & 4 \\
\hline Barrier only & 4 & 2 & 2 \\
\hline No Product & 5 & 3 & 0 \\
\hline
\end{tabular}

Methods Miniature diaphragm-like cervical barriers were manufactured and provided by ReProtect, Inc. BufferGel, previously found ineffective in this model when used alone, was also provided by ReProtect, Inc. Twenty-four pigtailed macaques were randomly assigned to one of three study arms: cervical barrier device alone; cervical barrier with BufferGel; or no barrier device and no gel (control arm). Eight animals were enrolled in each arm. Each macaque underwent baseline exam, product administration if applicable, cervical challenge with $\mathrm{C}$. trachomatis (E: $5 \times 105 \mathrm{IFU}$ ), and weekly follow-up exams for 5 weeks. Chlamydia challenge occurred within $30 \mathrm{~min}$ of baseline exams. Cervical barrier devices were removed from all test macaques $18 \mathrm{~h}$ after insertion. Each exam included cervicovaginal colposcopy, vaginal $\mathrm{pH}$ and cervical swabs for chlamydia detection (culture and NAAT: GenProbe Aptima Combo2). Blood for serum antibody testing was collected at baseline and weeks 2 though five post-inoculation.

Results Detection of chlamydial infection is detailed in the Abstract O4-S2.04 table 1 below. Colposcopic exams were comparable across each of the three test arms of the study, and thus did not detect toxic effects of the gel or the cervical barrier. Baseline vaginal $\mathrm{pH}$ was somewhat higher in the No Product arm than in either of the test arms. After chlamydial challenge and throughout the duration of the study, mean vaginal $\mathrm{pH}$ remained lowest in the barrier with BufferGel arm, but were similar (within $1 \mathrm{pH}$ unit) in all three study arms.

Conclusions In this pilot study, a cervical barrier used alone provided little or no protection, but the barrier used with BufferGel reduced transmission by $50 \%(p=0.08)$. These results should encourage further study of the ability of a cervical barrier combined with a microbicide to provide greater protection against sexually transmitted infections than either used alone.

\section{4-S2.05 MYD-88 DEFICIENT MICE SHOW EVIDENCE OF PRODUCTIVE T PALLIDUM INFECTION"}

doi:10.1136/sextrans-2011-050109.149

${ }^{1} \mathrm{D}$ Dunne, ${ }^{1} \mathrm{~A}$ Silver, ${ }^{1} \mathrm{~J}$ Fieber, ${ }^{2} \mathrm{C}$ Zeiss, ${ }^{1} \mathrm{E}$ Fikrig. ${ }^{1}$ Yale University School of Medicine, New Haven, USA; ${ }^{2}$ Yale School of Medicine, New Haven, USA

Background Syphilis rates are again increasing in the US and globally and are associated with HIV transmission and rising rates of congenital syphilis. Humans serve as the only natural reservoir of the causative agent, Treponema pallidum, and no in vitro cultivation systems are available. Progress towards a vaccine and a better understanding of immune response to syphilis has been hampered over the last half century by lack of a murine model. We hypothesised that previous attempts to establish $T$ pallidum infection in mice were unsuccessful because of rapid clearance of the organism by an intact innate immune response. Myd-88 serves as a common signalling molecule stimulated by most toll-like receptors TLRs; pattern recognition receptors of the innate immune system found on most innate immune cells, for example, monocytes, macrophages, and dentritic cells) and is responsible for downstream cytokine responses. We utilised mice bred to be Myd-88 deficient to test this hypothesis and to ascertain whether this mouse strain could be used to study persistent syphilis infection, immune response mechanisms, and eventually vaccine candidates.

Methods $T$ pallidum, Nicohols strain, was cultivated by intratesticular infection of New Zealand male rabbits housed at $62^{\circ} \mathrm{C}$ as per protocol. After 12 day incubation, T pallidum was extracted and the concentration adjusted to $7 \times 108$ organisms/ml. Myd- $88-/-$ mice and equally-aged C57BL/6 mice were inoculated intradermally, intraperitoneally, intravaginally or in testicles, and intrarectally each mouse receiving total dose of $1 \times 108$ organisms divided into the four body site aliquots). Mice were observed daily for signs or cutaneous disease and/or systemic illness and were sacrificed at day 10 and 21. DNA and RNA were extracted from skin, spleen, genitals, rectum, lymph nodes, spinal cord and blood for use in real-time quantitative PCR and RT-PCR, respectively. Corresponding tissue types were also evaluated by histopathology and immunohistochemical staining $T$ pallidum Ab, BioCare, Concord, California, USA). The experiment was repeated three times with variable number of mice per experiment.

Results A total of 18 Myd-88 mice and 19 C57BL/6 mice were infected. MyD-88 -/- mice were more likely than B6 control mice to have detectable RNA at day 10 and 21 (day 10, 12/35 sites (35\%) +RNA in Myd-88 -/- ; 3/35 sites (8.5\%) +RNA in WT. Day 21, $11 / 40$ sites $(27.5 \%)+$ RNA in Myd-88-/-; $3 / 40(7.5 \%)+$ RNA in B6). One experiment ongoing past 42 day post-infection reveals at day 42 sacrifice Myd-88 - /- mice with 6/15 sites (40\%) +RNA; B6 $0 / 15$ sites $(0 \%)+$ RNA. Histopathology revealed mild to moderate inflammation in MyD88-/- mice and demonstrable organisms by IHC Abstract O4-S2.05 figure 1).

Conclusion These preliminary experiments suggest that the immune recognition impairment caused by deleting Myd88 signalling protein results in productive and longer-lasting $T$ pallidum infection in this

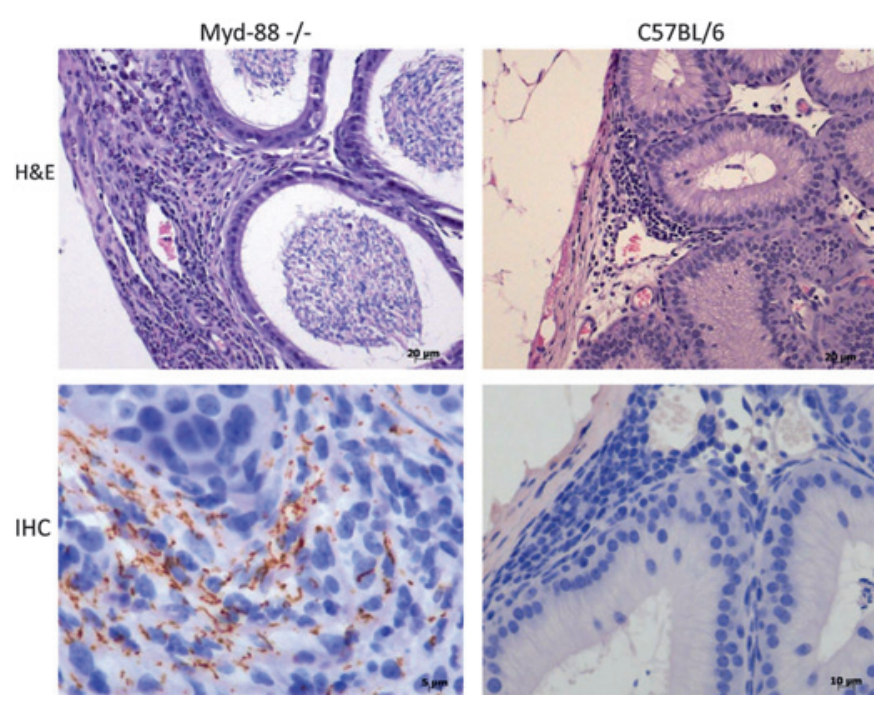

Abstract 04-S2.05 Figure 1 Day 21 Post T.pallidum infection Epididymis. 